\title{
Ways of Thanking in Mandarin Chinese and English
}

\author{
Kai-ting Chuang, Shelley Ching-yu Hsieh \\ National Cheng Kung University, Tainan, Taiwan
}

This is a corpus-based study investigating ways of thanking in Mandarin Chinese and English. Sociolinguistic and semantic perspectives are adopted to observe the data, which are taken from the Academia Sinica Balanced Corpus of Modern Chinese and the Lancaster BNC web. The theoretical background is supported by Wichmann's (2004) concept of the position of the word, and Cheng's (2010) compliment response and thanking strategies. The results show that: (1) Mandarin Chinese xie4xie4 (谢谢 “thank”) carries more of the compliment response strategy and has more varied positions within a sentence than the items gan3xie4 (感谢 “thank”, “thanks”, and “thank you”); (2) Regarding the compliment response strategy, Mandarin Chinese xie4xie4 has a more extended compliment response strategy, except for the simple thanking strategy, and also includes the response strategies of rejection or appreciation. Mandarin Chinese gan3xie4 manifested the tendency on the compliment response strategy of thanking. The two different response strategies between xie4xie4 and gan3xie4. On the other hand, the category of “accept" and strategy of "thanking” are both the most representative uses for thank you and thanks among the utterances extracted from the corpus; and (3) With regard to cultural values, attitudes to life and social behaviors play an important role in explaining the linguistic phenomena in both languages. We hope the findings of the present study can promote communication between Mandarin and English speaking people, as well as help L2 (second language) students with Mandarin Chinese and English learning.

Keywords: corpus linguistics, ways of thanking, compliment response, Mandarin Chinese, English

\section{Introduction}

A large body of research has investigated the way we communicate with people from different cultural backgrounds, focusing on actions such as requesting, thanking, apologizinging, and closing an interaction (Wolfson, 1989; Holmes \& Brown, 1987; Borkin \& Reinhart, 1978; Eisenstein \& Bodman, 1986). Zhu and Bao (2010) noted the effects that cultural differences have on the language use, and how gratitude is expressed is also influence by this.

The present study aims at investigating actual utterances with data taken from corpora to analyze ways of thanking and syntactic structures and response strategies in Mandarin Chinese and English. The sources of the data are the Academia Sinica Balanced Corpus of Modern Chinese (hereafter Sinica Corpus) and the Lancaster $B N C$ web (hereafter Lancaster BNC).

This study was designed to explore the syntactic structures and the response strategies related to thanking

Kai-ting Chuang, graduate student at Department of Foreign Language and Literature, National Cheng Kung University. Shelley Ching-yu Hsieh, professor, Department of Foreign Language and Literature, National Cheng Kung University. 
expressions in the two focal languages, as well as to examine whether any of differences that are found are due to the varied cultures. In order to achieve the goals of this research, the position of the word "please" is categorized based on various criteria (Wichmann, 2004), and various categories of compliments response strategies (Holmes, $1988,1993)$ are adopted to help classify the thanking expressions. The basic structure of this paper is as follows: (1) introduction; (2) literature review; (3) methodology, with the data source and analytic framework; (4) data analysis, where the syntactic structures and compliment response strategies of thanking are presented, along with a comparison between Mandarin Chinese and English; (5) the different attitudes to life in Western and Eastern cultures are discussed; and finally (6) the conclusion of this work is presented.

\section{Literature Review}

People are more likely to accomplish their communication goals if their interactions are appropriate to the context they are situated in (Cheng, 2010), and the speech act of thanking has drawn the particular attention of scholars with regard to this. Chen (1993) noted the different compliment responses used by people in China and the UK, and responses to "thank you" can vary from culture to culture. Different culture may respond differently to other's compliment. In additon, the way that people express thanks is related to the different situations of a compliment. Chen (1993) quoted Holmes (1986) and said that "A compliment is a speech act which explicitly or implicitly attributes credit to someone other than the speaker, usually the person addressed, for some 'good' (possession, characteristic, skill, etc.) which is positively valued by the speaker and the hearer” (p. 446). Holmes (1998, p. 448) also claimed that a compliment can be face threatening, as one implication of a compliment is the speaker's envy of the person they are talking to.

Cheng (2010) also indicated that compliment responses serve have a bonding function in personal relationships. Chen's (1993, p. 64) study of the compliment responses made by Chinese and English native speakers found that the former tended to reject compliments, while the latter generally accepted and appreciated them. Yu (2004) further stated that the use of compliment responses in Chinese and English speech communities vary both pragmatically and culturally due to the prevailing social norms.

Some studies have used a politeness principle (Leech, 1983, p. 135) that to examine the different forms of communication in Western and Chinese cultural contexts (Zhu \& Bao, 2010).

Pomerantz (1978, pp. 81-82) claimed that the two general conditions which govern the act of responding to a compliment are agreeing with the speaker and avoiding self-praise. These studies show that the most basic and the common response to compliments is to say "thank you", although in some situations people want to avoid self-praise by expressing disagreement.

These earlier works have shed light on the significance of compliments and the way of thanks is expressed in the communication, especially in cross-cultural communication. While considerable efforst have been made to examine how thanks are expressed by analyzing the linguistic structures or the language used in such contexts, relatively few studies compare the semantic and syntactic structures of thanking in Mandarin Chinese and English, and this is the goal of the present study.

Based on observations using corpus data, the present study aims at comparing the syntactic and pragmatic aspects of thanking in two languages, and draws out the cultural differences these reveal with regard to attitudes toward life, social behaviors, and cultural values. 


\section{Methodology}

This section presents the data collection process and analytic framework used in this study, with the data drawn from the Lancaster BNC and Sinica Corpus. In addition, the process of analysis used for the syntactic structures and the compliment response strategies will be introduced.

\section{Data Collection}

The data are obtained from the Lancaster BNC and the Sinica Corpus. The Lancaster BNC is a free and open source corpus that consists of 100 million words in the form of both written and spoken texts. This collection can help with regard to some of the main issues in corpus linguistics, such as collocations, key words, and the categorization of concordance lines. The query options in the Lancaster BNC are divided into spoken and written restrictions, and standard queries.

Sinica Corpus contains about five million words covering many different topics and genres, such as literature, life, social science, philosophy, and arts. Using these two collections, we collected 1,074 pieces of raw data containing examples of Mandarin Chinese xie4xie4 (谢谢 “thank”) and gan3xie4 (感谢 “thank”), and 10,000 example of English thank you and thanks, as shown in Table 1.

Table 1

The Raw Data From Sinica Corpus and Lancaster BNC

\begin{tabular}{llcc}
\hline Language & Raw data & Amount & Total \\
\hline Mandarin Chinese & xie4xie4 (谢谢 “thank”) & 832 & 1,074 \\
& gan3xie4 (感谢 “thank”) & 242 & \multirow{2}{*}{10,000} \\
\hline English & thank you & 5,000 & 5,000 \\
& thanks & & \\
\hline
\end{tabular}

\section{Analytic Framework}

To compare the syntactic structures and the compliment response strategies in Mandarin Chinese and English thanking expressions, this study adopts Wichmann's (2004) syntactic aspects for please-requests, which states that the word please can be categorized based on its position, such as in the initial, medial, final, and other place. For example, in English, a sentence like I'm not too bad, thank you has "thank you” placed in the final position of the sentence. In a Mandarin Chinese sentence like wǒmen xiān hē diăn ēr jiǔ hăo bù hăo (“我们先喝 点儿酒, 好不好? Would you like some drink?”) xiè xie wǒ bù hējiǔ (“谢谢, 我不喝酒。No, thanks. I don't drink."), xie4xie4 ("thanks") is located in the initial position. In the present study, xie4xie4 ("thank"), gan3xie4 ("thank"), thanks, and thank you will be categorized into tokens that take the initial, medial, or final positions according to their syntactic positions in the utterances being examined. In addition to identifying the compliment responses, the macro level of thanking and thanking expressions will also be investigated in this work.

Holmes $(1986,1993)$ examined various compliment response strategies, and proposed three macro level ones: accept, reject, and evade. Cheng (2010) categorized six major types of thanking, and identified thanking, appreciation, non-gratitude, combination, thanking a third person , and formal speech (see Table 2). For example, “Thanks, he put his cup away" which can be seen as a reject strategy according to Holmes (1986, 1993), while according to Cheng (2010) it is a non-gratitude type of thanking, which means to rejecting an offer from somebody else. 
Table 2

The Six Major Categories of Thanking Expressions (Cheng, 2010, pp. 262-264)

\begin{tabular}{|c|c|}
\hline Categories of thanking expressions & Examples \\
\hline $\begin{array}{l}\text { Thanking: (a) simple thanking } \\
\text { (b) elaborated thanking } \\
\text { (a) thank you/thank } \\
\text { (b1) Thanking + one intensifier } \\
\text { (b2) Thanking + two intensifiers } \\
\text { (b3) Thanking for reason } \\
\text { (b4) Thanking + intensifier + reason }\end{array}$ & $\begin{array}{l}\text { (a) Thanks. } \\
\text { (b1) Thank you very much. } \\
\text { (b2) Thank you very much indeed. } \\
\text { (b3) Thank you for your consideration and your attention to all of } \\
\text { the things that we brought forward. } \\
\text { (b4) Thank you very much for showing us your home. }\end{array}$ \\
\hline $\begin{array}{l}\text { Appreciation: } \\
\text { By appreciation and adding intensifier } \\
\text { By showing appreciation and stating reason } \\
\text { By showing appreciation and adding intensifier and the reason }\end{array}$ & $\begin{array}{l}\text { (1) It's much appreciated. } \\
\text { (2) If you don't want to be public about it and will tell me later, I } \\
\text { would appreciate that. } \\
\text { (3) We would really appreciate it if you could come. }\end{array}$ \\
\hline $\begin{array}{l}\text { Non-gratitude: } \\
\text { By showing relief } \\
\text { By rejecting an offer } \\
\text { By showing politeness, greeting, and conversation ending } \\
\end{array}$ & $\begin{array}{l}\text { (1) Thank goodness. } \\
\text { (2) I can manage on my own. Thank you. } \\
\text { (3) Have a nice day. Thank you. You, too. }\end{array}$ \\
\hline $\begin{array}{l}\text { Combination: } \\
\text { By thanking and stating the reason } \\
\text { By thanking and adding intensifier and reason } \\
\end{array}$ & $\begin{array}{l}\text { (1) Thank you dear. Very kind of you. } \\
\text { (2) Thank you very much. I am sorry to bother you. }\end{array}$ \\
\hline $\begin{array}{l}\text { Thanking a third person: } \\
\text { By thanking a third person and stating the reason } \\
\text { By thanking a third person and adding intensifier and the reason }\end{array}$ & $\begin{array}{l}\text { (1) It falls uh to me on your behalf uh to thank uh, Gary Glick for } \\
\text { a talk. } \\
\text { (2) Many thanks to Eunice and Matt for lots of hard work and for } \\
\text { really making a big leap forward and for going from where we } \\
\text { were in Chincago in terms of text to where we are today. }\end{array}$ \\
\hline $\begin{array}{l}\text { Formal speech: } \\
\text { By formally thanking and stating the reason } \\
\text { By formally thanking a third person } \\
\text { By formally thanking a third person and stating the reason }\end{array}$ & $\begin{array}{l}\text { (1) I thank you, Pam, because I think the committee's done a } \\
\text { great job. } \\
\text { (2) The committee would like to publicly thank Garland Hershey } \\
\text { and Dick McCormick and their offices. } \\
\text { (3) We would like to thank Garland Hershey and Dick } \\
\text { McCormick and especially the staffs in their offices for all the } \\
\text { help they have given us this past year in gathering the data for the } \\
\text { glass ceiling study that we are currently engaged in, and } \\
\text { hopefully next year we will have in our report the conclusions } \\
\text { from that study. }\end{array}$ \\
\hline
\end{tabular}

In sum, the syntactic analysis on position (Wichmann, 2004), the strategy of compliment response (Holmes, 1986, 1993), and the six types of thanking expressions (Cheng, 2010) will all be applied in the analysis that is carried out below.

\section{Analysis and Finding}

Section 4.1 gives the syntactic structure of thanking, while section 4.2 presents the response strategy of thanking. Finally, section 4.3 compares thanking in Mandarin Chinese and English.

\section{The Syntactic Structure of Thanking}

Based on the method presented in Wichmann (2004), our data are categorized into initial, medial, or final sentential positions. In Example 1 below, xie4xie4 ("thank") appears in the initial place of the response, which is a polite rejection of an invitation. At the beginning, the speaker invites the hearer to stay, but the hearer responds with by using xie4xie4 to express his gratitude for the invitation, although he declines it.

Example (1) Zai4wo3men0zhe4er0chi1yi4dian3er0dong1xi1, hao3bu4hao3?

Xie4xie4! xie4xie4! nin2tai4ke4qi4, wo3men0hai2you3bie2de0shi4qing2 
在我们这儿吃一点儿东西, 好不好?

谢谢! 谢谢! 您太客气, 我们还有别的事情。

Would you like to eat something here?

No, Thanks. You are welcome. We still have something to do.

Table 3 presents the results of the analysis of the spoken data for thanks, thank you, xie4xie4 ("thank"), and gan 3xie4 ("thank") with regard to their positions within declarative sentences.

Table 3

The Positions of Thanking Expressions in Declarative Sentences

\begin{tabular}{|l|l|l|l|l|l|l|l|}
\hline xie4xie4 (谢谢 “thank”) & Position & & & gan3xie4 (感谢“thank”) & Position & & \\
\hline & Initial & Medial & Final & & Initial & Medial & Final \\
\hline Declarative & & & $40 \%$ & Declarative & $85 \%$ & & \\
\hline Declarative & & $45 \%$ & & Declarative & & $10 \%$ & \\
\hline Declarative & $15 \%$ & & & Declarative & & & $5 \%$ \\
\hline Total & $100 \%$ & & & Total & $100 \%$ & & \\
\hline English thanks & & & & English thank you & & & \\
\hline & Initial & Medial & Final & & Initial & Medial & Final \\
\hline Declarative & & & $15 \%$ & Declarative & $55 \%$ & & \\
\hline Declarative & & $80 \%$ & & Declarative & & $15 \%$ & \\
\hline Declarative & $5 \%$ & & & Declarative & & & $30 \%$ \\
\hline Total & $100 \%$ & & & Total & $100 \%$ & & \\
\hline
\end{tabular}

Table 3 shows that xie4xie4 appears both in the medial or final positions of a declarative sentence in Mandarin Chinese. However, the results for thanks are different. Thank you is frequently occurred in the initial position, while thanks is most often in the medial one. It is worth noting that in $85 \%$ of the examples gan $3 x i e 4$ ("thank") is in the initial position. Comparing the two thanking expressions of xie4xie4 ("thank") and gan3xie4 ("thank"), Table 3 shows slight differences between the numbers of medial and final positions, while gan $3 x i e 4$ ("thank") mostly appears in the initial position.

\section{The Compliment Response Strategy of Thanking}

The concept of compliment responses from Holmes and the six major types of thanking categorization are applied in this section to examine the compliment response strategies used with thanking expressions in Mandarin Chinese and English.

Based on criteria in Holmes (1988, 1993) and Cheng (2010), the results for xie4xie4, gan3xie4 (“thank”), thanks, and thank you show some significant variations. Example 2 illustrates the strategy of rejection with regard to an invitation to have a drink. This example falls into the macro level "reject" compliment response and follows the thanking category "non-gratitude". The example refers to the strategy of reject belongs to the categorization of "non-gratitude".

Example (2) Wo3men0 xian1 he1dian3er0jiu3, hao3bu4hao3?

Xie4xie4, wo3bu4he1jiu3

我们先喝点儿酒, 好不好?

谢谢，我不喝酒。

Would you like a drink?

No, Thanks. I don’t drink. 
Example (3) Zui4hou4, wo3men0gan3xie4zhong1shi2wan 3bao4dui4jin1tian1yan2tao3hui4de0zan4zhu4. ye3zai4du4dui4 ge4wei4fa1qi3ren2de0xin1lao2, biao3shi4xie4yi4. ye3gan3xie4jiao4yu4bu4gu4 wen4shi4he2zhong1shi2 wan3bao4de0 zhi1chi2zan4zhu4. 最后，我们感谢中时晚报对今天研讨会的赞助。 也再度对各位发起人的辛劳，表示谢意。 也感谢教育部顾问室和中时晚报的支持赞助。

Finally, we appreciated for the sponsorship of our conference that we received from China Times.

Also, I want to express my gratitude for the hard work of every initiator and the sponsorship of the China Times and the Minister of Education consultant group.

Example (4) Thanks, Roger, but I have to look for someone.

Example (5) But Steffi fans will like to express their thanks and wish her well.

Examples 3-5 all represent different types of thanking. Example 3 shows the acceptance strategy of compliment response, and belongs to the category of formal speech. Formal speech can be identified by formally thanking and stating the reason in the utterance, and this is usually announced by overt subject, such as "I" or "we". Example 4 refers to the category of combination, in that the thanking is followed by a reason, and this can also be a strategy of evasion, which is not as direct as a rejection. On the other hand, Example 5 shows the thanks being directed towards a third person (Steffi fans), and thus it is an example of the compliment response strategy of acceptance.

Tables 4-5 show that the most frequently adopted compliment response strategy in both Mandarin Chinese and English languages was acceptance, which accounted for $72 \%$ of the uses of xie4xie4 ("thank") and thanks, $100 \%$ of gan 3xie4 ("thank"), and 68\% of thank you. The other two compliment response strategies, rejection and evasion, do not show any significant differences with regard to the frequency of xie4xie4 ("thank") and thank you. As seen in Table 4, Mandarin Chinese xie4xie4 ("thank") and English thank you toward the application in the strategy of rejection and evasion do not reveal the differences in the percentage distribution. In Table 4, English thank you and thanks gain more proportions in the application of the strategy rejection than evasion. Nevertheless, Mandarin Chinese xie4xie4 ("thank") received more numbers of proportion in the application of strategy evasion. In the research of Holmes (1993) about New Zealander's response to compliment, the most common strategy is accept, then follows the strategy of evasion and rejection. In our data, thank you and thanks tend to be frequently used as the strategy of rejection while xie4xie4 ("thank") inclined to be adopted as the strategy of evasion. It is noteworthy that the compliment response strategy of gan3xie4 ("thank") is always used with the acceptance strategy in the corpora data examined in the present study. This means that gan3xie4 ("thank") is always used to accept a compliment and also used for acceptance quite often.

In addition, gan3xie4 ("thank") and thanks both have the same frequencies in the "formal speech" category. In most of the sentences, gan 3xie4 ("thank") refers to the "thanking” category. Table 5 shows that thank you and thanks differ with regard to the categories of "non-gratitude" and "appreciation". There are no examples of thanks in the category of "non-gratitude", while thank you has no examples in the category of "appreciation". In addition, neither thanks no thank you are in the category of "combination", with this category containing thanking plus reason or intensifier when expressing gratitude in a sentence. 
Table 4

The Compliment Response Strategy of Thanking

\begin{tabular}{|l|l|l|l|}
\hline \multicolumn{1}{|c|}{ xie4xie4 (“thank") } & Percentage (\%) & \multicolumn{1}{c|}{ gan3xie4 (“thank”) } & Percentage (\%) \\
\hline Macro level strategies of compliment response & & Macro level strategies of compliment response & \\
\hline Accept & 72 & Accept & 100 \\
\hline Reject & 12 & Reject & \\
\hline Evade & 16 & Evade & English thank you \\
\hline \multicolumn{1}{|c|}{ English thanks } & & & \\
\hline Macro level strategies of compliments response & & Macro level strategies of compliments response & \\
\hline Accept & 72 & Accept & 68 \\
\hline Reject & 24 & Reject & 20 \\
\hline Evade & 4 & Evade & 12 \\
\hline Total & 100 & Total & 100 \\
\hline
\end{tabular}

Table 5

The Six Major Types of Thanking Expressions

\begin{tabular}{|c|c|c|c|}
\hline xie4xie4 ("thank") & Percentage (\%) & gan3xie4 (“thank”) & Percentage (\%) \\
\hline Thanking & 20 & Thanking & 48 \\
\hline Appreciation & 8 & Appreciation & +2 \\
\hline Non-gratitude & 44 & Non-gratitude & 7 \\
\hline Combination & 8 & Combination & 7 \\
\hline Thanking of a third person & - & Thanking of a third person & 28 \\
\hline Formal speech & 20 & Formal speech & 24 \\
\hline English thanks & & English thank you & \\
\hline Six major types of thanking expressions & & Six major types of thanking expressions & \\
\hline Thanking & 32 & Thanking & 52 \\
\hline Appreciation & 28 & Appreciation & 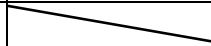 \\
\hline Non-gratitude & & Non-gratitude & 36 \\
\hline Combination & 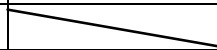 & Combination & 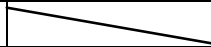 \\
\hline Thanking of a third person & 16 & Thanking of a third person & 8 \\
\hline Formal speech & 24 & Formal speech & 4 \\
\hline Total & 100 & Total & 100 \\
\hline
\end{tabular}

\section{A Comparison of Mandarin Chinese and English}

This section compares the differences between Mandarin Chinese and English with regard to the sentence structure and compliment response strategy of thanking.

First of all, the syntactic distribution of xie4xie4 ("thank"), gan3xie4 ("thank") in the declarative sentences show differences in their positions in a sentence. For xie4xie4 ("thank"), the results show the average proportion from Table 3 in both the middle and final positions of a declarative sentence. However, for gan3xie4 ("thank") appears in the initial place of and occupied $85 \%$ in declarative sentence. On the other hand, the English data reveal the unobvious proportion after the comparison in initial, medial, and final positions in declarative sentences with thank you, while thanks most often appears in the middle position in declarative sentences. Thank you and thanks unlike xie4xie4 and gan3xie4 reveal the difference on position.

Secondly, the results of comparing the compliment response strategy using xie4xie4 ("thank"), gan3xie4 (“thank"), thanks, and thank you are shown in Tables 4-5. "Accept” strategy is widely used in xie4xie4 ("thank") 
and gan3xie4 ("thank"). However, the results also reveal that xie4xie4 ("thank") is used most often for the non-gratitude compliment response strategy. However, gan3xie4 (“thank”) is always used as the strategy of accept (100\%). In English, the strategy of accept and categorization of thanking of thank you and thanks show quite similar results.

A comparison of the thanking expression compliment response strategy in Mandarin Chinese and English shows that gan3xie4 ("thank"), thank you, and thanks are all very often used to accept things, and thus belong to the compliment response strategy of thanking. On the other hand, Mandarin xie4xie4 ("thank") is often used for compliment response strategy of "non-gratitude", which shows that this expression can be used for the strategies of rejection and evasion, as well as acceptance.

\section{Discussion}

This section explores different attitudes to life in Chinese and English-speaking cultures based on the different syntactic structures and compliment response strategies used with thanking expressions, as discussed above.

Xie4xie4 ("thank") not only expresses the feeling of gratitude toward somebody, but also shows respect and appreciation to the interlocutors or matters being discussed present. It is also possible to reject something or somebody by saying xie4xie4 ("thank") as a polite strategy. Chinese includes Taiwanese people are conservative when it comes to refusing things, and because of its Confucian education system people are very concerned about hurting other people's feelings. The syntactic positions of xie4xie4 ("thank") which only mentioned in Chinese data and thank you within sentences have some relations with the cultures.

In addition, the way gratitude is expressed can also be related to a person's attitude to life. According to the position in a sentence comparison, the present study aims at explaining the different expression of gratitude. Chinese people tend to put xie4xie4 ("thank") in the middle or final position of sentence to be polite and respond to the compliments they are receiving. This situation can be compared to the use of the English thank you, which often appears in the initial position of a declarative sentence. In Western culture, like that of native speaker in English, people tend to express their feelings and talk directly, and thus it is not surprising that we found more examples of thank you in the initial position in our corpus data. Compares to Chinese people, native speaker in English are more direct in their attitudes toward life.

Furthermore, the analysis of the compliment responses carried out in this work demonstrates the different uses of the strategies of accept, reject, and evade. Gan3xie4 ("thank") is always used for the compliment response strategy of accept in Table 4. Compared to xie4xie4 ("thank"), gan3xie4 ("thank") is more limited on the strategy unlike xie4xie4 in its use of compliment response strategy. Chinese culture tends to adopt various degrees of gratitude words to represent different degree of thanking. The Chinese gratitude word gan3xie4 (“thank") is more often used as a form of emphasis with the compliment response strategy than the ordinary use of xie4xie4 ("thank"). There are also many words expressed different degree of gratitude in Chinese such as, gan3en1 (感恩 “gratitude”) or gan3ji1 (感激) is used to indicated a deeper sense of gratitude. However, thank you and thanks do not represent the different degrees of gratitude, except when other terms are added, such as very much or a lot after them.

To sum up, the syntactic position helps to explain the direct and indirect expression of compliment. In addition, the compliment response strategy in Chinese has more terms that can be used to express different degrees of gratitude than English. Therefore, the analysis of the syntactic distribution and compliment response 
strategies carried out in this work can not only improve our linguistic understanding of thanking, but also reveal certain things about cultural differences.

\section{Conclusions}

This study examined the syntactic and semantic aspects of thanking expressions in Mandarin Chinese and English in order to better understand the characteristics of the syntactic and semantic distinctions and cultural values expressed in these two languages.

With regard to the compliment response strategy, xie4xie4 ("thank") is used for an extended compliment response strategy that goes beyond simple thanking, and can include the response strategies of rejection and appreciation. Gan3xie4 ("thank") is most often used for the compliment response strategy of thanking. On the other hand, the English expressions thank you and thanks appeared in most of the examples with the compliment response of acceptance and the strategy of thanking. Regarding the syntactic analysis of gratitude expressions, xie4xie4 ("thank") is located at the medial or final positions in most of the declarative sentences examined in this work, while the English thank you and thanks are more often in the initial or middle positions. These results can be explained by the different attitudes to life in English and Mandarin Chinese people.

This study of gratitude expressions reveals the connections between language patterns and culture. Further research may explore the pragmatic functions and cognitive processes related to gratitude in different languages. More understanding of gratitude expressions can not only help people to achieve more effective communication, but can also help to create more harmonious relationships among different cultures.

\section{References}

Borkin, A., \& Reinhart, S. M. (1978). Excuse me and I’m sorry. TESOL Quarterly, 12(1), 57-69.

Chen, R. (1993). Responding to compliments: A contrastive study of politeness strategies between American English and Chinese speakers. Journal of Pragmatics, 20(1), 49-75.

Cheng, S. W. (2010). A corpus-based approach to the study of speech act of thanking. Concentric: Studies in Linguistics, 36(2), 257-274.

Eisenstein, M., \& Bodman, J. W. (1986). "I very appreciate”: Expressions of gratitude by native and non-native speakers of American English. Applied Linguistics, 7(2), 167-185.

Herbert, R. K. (1986). Say “thank you”-Or something. American Speech, 61(1), 76-88.

Holmes, J. (1986). Compliments and compliment responses in New Zealand English. Anthropological Linguistics, 28(4), $485-508$.

Holmes, J. (1988). Paying compliments: A sex-preferential politeness strategy. Journal of Pragmatics, 12(4), 445-465.

Holmes, J. (1993). New Zealand women are good to talk to: An analysis of politeness strategies in interaction. Journal of Pragmatics, 20(2), 91-116.

Holmes, J., \& Brown, D. F. (1987). Teachers and students learning about compliments. TESOL Quarterly, 21(3), 523-546.

Leech, G. N. (1983). Principles of pragmatics (Vol. 1). London: Longman.

Pomerantz, A. (1978). Compliment responses: Notes on the co-operation of multiple constraints. Studies in the organization of conversational interaction (pp. 79-112). New York: Academic Press.

Wichmann, A. (2004). The prosody of please-requests: A corpus based approach. Journal of Pragmatics, 36(9), $1521-1549$.

Wolfson, N. (1989). The social dynamics of native and nonnative variation in complimenting behavior. In M. R. Eisenstein (Ed.), The dynamic interlanguage: Empirical studies in second language variation (pp. 219-236). New York: Plenum Press.

$\mathrm{Yu}$, M. C. (2004). Interlinguistic variation and similarity in second language speech act behavior. The Modern Language Journal, $88(1), 102-119$.

Zhu, J., \& Bao, Y. (2010). The pragmatic comparison of Chinese and western "politeness” in cross-cultural communication. Journal of Language Teaching and Research, 1(6), 848-851. 